\title{
Immunoglobulin G4-Related Disease in the Head and Neck: Two Case Reports and Literature Review
}

\author{
Morteza MAZLOUM FARSI BAF, ${ }^{1}$ Maryam SAHEBARI, ${ }^{1}$ Kamran KHAZAENI, $^{2}$ Zahra REZAIEYAZDI ${ }^{1}$ \\ ${ }^{1}$ Rheumatic Diseases Research Center (RDRC), Ghaem Hospital, Faculty of Medicine, \\ Mashhad University of Medical Sciences, Mashhad, Iran \\ ${ }^{2}$ Sinus and Surgical Endoscopic Research Center, Ghaem Hospital, Faculty of Medicine, \\ Mashhad University of Medical Sciences, Mashhad, Iran
}

\begin{abstract}
Immunoglobulin G4-related disease (IgG4-related disease) is a fibroinflammatory disorder defined by elevated serum lgG4 level and plasma cells tissue infiltration accompanied by fibrosis or sclerosis. In this article, we report two patients of IgG4 syndrome: a 60-year-old male with complaints of nasal congestion, rhinitis, anosmia and hearing loss, and a 28 -year-old female with bilateral orbital and parotid gland swelling. Histopathological findings and high serum IgG4 level confirmed diagnosis of IgG4-related disease. Both patients were treated successfully with glucocorticoids and methotrexate. Herein, we also review clinical presentations of IgG4-related disease in head and neck, and related diagnostic and therapeutic approach.

Keywords: Immunoglobulin G4-related disease; Mikulicz's syndrome; Sjögren's disease.
\end{abstract}

Immunoglobulin G4-related disease (IgG4-related disease) is a fibroinflammatory disorder. It was considered as Mikulicz's syndrome or a kind of Sjögren's syndrome (SS) in the past decades; however, now it is recognized as a novel entity which can affect various organs. The unique pathological features of the disease are systemic inflammation, lymphoplasmocytic infiltration with IgG4-positive plasma cells and fibrosis that often but not always are accompanied by elevated serum IgG4 concentrations. ${ }^{1-4}$

The entity of "IgG4-related autoimmune disease" was introduced by Kamisawa et al. ${ }^{5}$ for the first time 10 years ago. Since then, many disorders which were previously considered as organ specific have been included in the spectrum of IgG4-related disease. ${ }^{6}$

Immunoglobulin $\mathrm{G} 4$ syndrome involves various organs such as pancreas, bile ducts, salivary glands, periorbital tissues, kidneys, lungs, lymph nodes, meninges, aorta, breast, prostate, thyroid gland, retroperitoneum, pericardium, and skin. ${ }^{7-11}$ It may cause various clinical presentations based on involved organ such as sclerosing pancreatitis, sclerosing cholangitis, prostatitis, tubulointerstitial nephritis, interstitial pneumonia, and enlargement of salivary glands..$^{3,4}$ Early diagnosis is important to prevent tissue damage, fibrosis, and sclerosing.

Up to now, various patients of IgG4-related disease with different organ involvements and various manifestations have been reported. ${ }^{12,13}$ In this article, we present two patients of IgG4-related disease with head and neck involvement, and review the related literature.

\section{CASE REPORT}

Case 1- A 60-year-old male admitted to our clinic with complaints of nasal congestion, rhinitis, 
anosmia, and hearing loss accompanied by some episodes of fever without cough or any other remarkable symptoms that had been aggravated during the last three months. Except for a history of dry eye, he did not mention any special disorder in himself or his family. Physical examination showed generalized muscle weakness.

With primary diagnoses of sinusitis and ear infection, some paraclinical evaluations were requested. Computed tomography scans of paranasal sinuses showed opacity and widening of ostiomeatal complex but his ears were normal. A few days later, bilateral parotid and submandibular salivary glands swelled. Serological tests and lymph node biopsies were performed to clarify the situation. Laboratory examination revealed negative antinuclear antibodies profile, increased erythrocyte sedimentation rate $(80 \mathrm{~mm} / \mathrm{h})$, and high $\mathrm{IgG} 4$ titer (405 mg/dL) (normal 6.2-112 mg/dL). Other serologic examinations for infectious diseases such as cytomegalovirus, herpes simplex virus, Epstein-Barr virus, mycoplasma, and hepatitis $B$ and $C$ viruses were negative but serum IgG4 was elevated. Abdominal and chest computed tomography scans were unremarkable. Histopathological examination of salivary glands showed focuses of numerous lymphoid follicles with germinal centers, extensive fibrosis, and abundant IgG4+ plasma cell infiltration. Thus the diagnosis of IgG4-related disease was confirmed and treatment was started with steroid. To reduce steroid-induced adverse effects, methotrexate was added to the treatment regimen. After three months of follow-up, patient's symptoms improved significantly. One year after the end of treatment, patient was healthy and symptom free.

Case 2- A 28-year-old female referred to our clinic with bilateral proptosis and parotid swelling from three months ago. She had a history of bilateral parotid swelling from six months ago, as well as chronic sinusitis and mild asthma. Her problem started three years ago with bilateral upper eyelid swelling that improved by using prednisolone. However, discontinuation of treatment due to pregnancy caused symptoms to relapse at second trimester of pregnancy.

In physical examination, visual acuity and neurologic examination were normal. There was no eye or mouth dryness. Hyperpigmented patchy lesions were observed on left leg and both hands, and bilateral eye and neck swellings were detected as well.

Chest-X-ray and abdominal ultrasound were unremarkable. Chest computed tomography scan showed right hilar lymphadenopathy and local plural thickening in posterior part of left hemithorax. Head and neck magnetic resonance imaging (MRI) indicated multiple hypointense signal foci in $T_{2}$ in orbital muscles and cervical lymph nodes (Figure 1). Sputum smear and culture were negative for acid fast bacilli. Thyroid function tests and other biochemical parameters were normal.
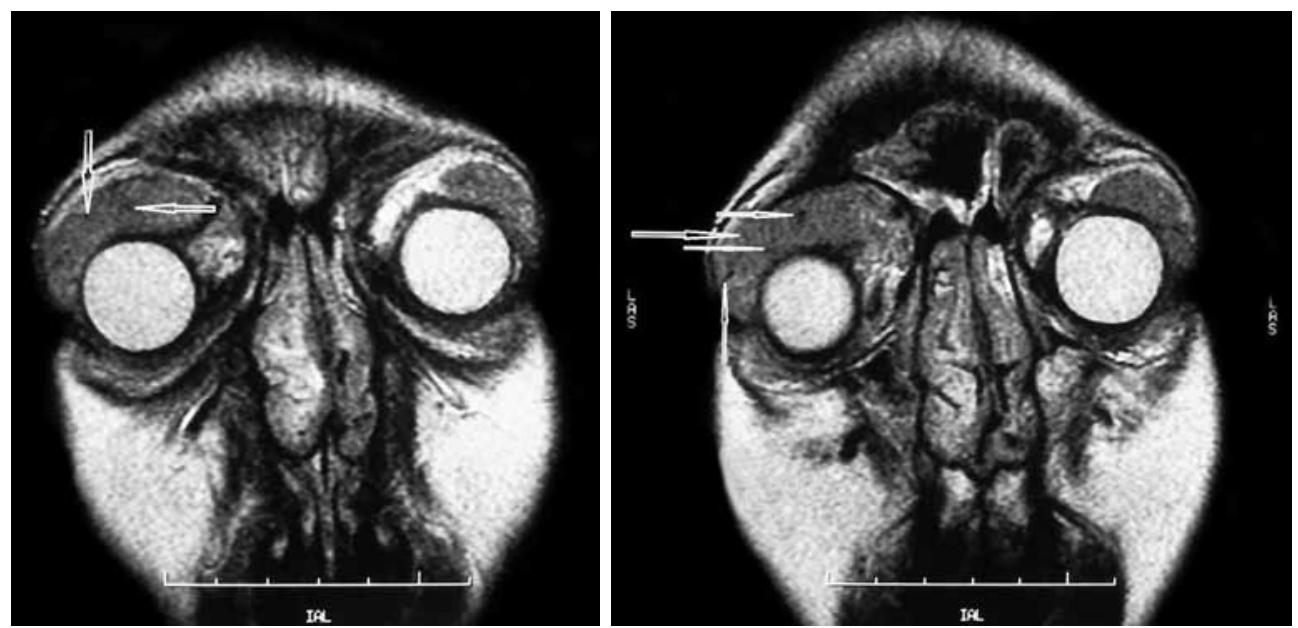

Figure 1. Orbital magnetic resonance imaging with hypointense signal foci in $T_{2}$ in the orbital muscles. 


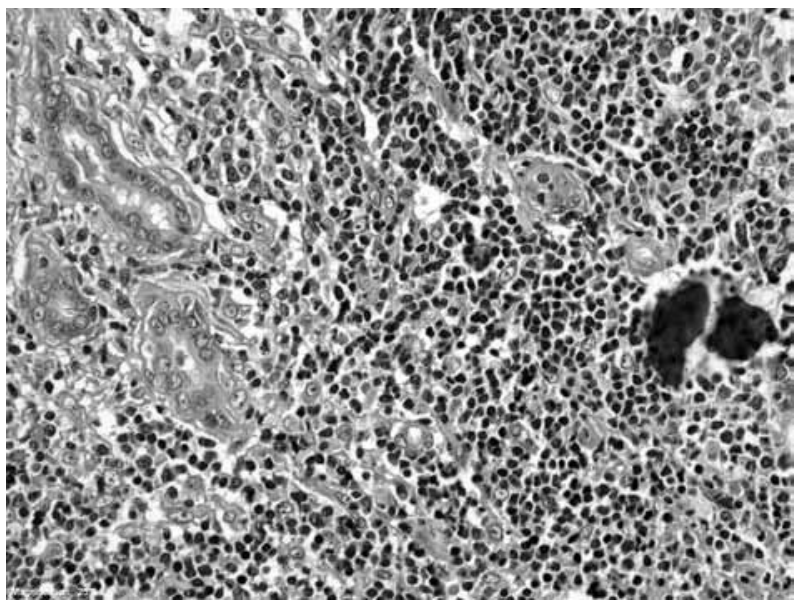

Figure 2. Orbital mass histopathology showing lymphoplasmacytic infiltration with IgG4-positive plasma cells (H-E x 400).

Orbital mass biopsy was performed and histopathological exam showed numerous lymphoid follicles with germinal centers, fibroid bundles and infiltration of IgG4+ plasma cells and eosinophils in lacrimal gland (Figure 2). Results of serologic assays including anti nuclear antibody profile and complement $\mathrm{C} 3, \mathrm{C} 4$ and $\mathrm{CH} 50$ were in normal ranges but serum $\mathrm{IgG} 4$ level was elevated $(817 \mathrm{mg} / \mathrm{dL}$ ) (normal range: 6.2-112 mg/dL).

Diagnosis of IgG4-related disease was made based on histopathological findings and high serum concentration of IgG4. Treatment was started with high dose of prednisolone $(1 \mathrm{mg} / \mathrm{kg}$ ) and then methotrexate was added to reduce steroid-induced side effects. As the patient was cushingoid, the response to steroid therapy was not too favorable and her blood glucose increased by therapy. We were unable to stop the treatment since steroid tapering and reducing steroid dosage caused relapse of symptoms. Patient may be a candidate for other new therapies like $\mathrm{B}$ cell depletion therapy with rituximab.

\section{DISCUSSION}

Immunoglobulin G4-related disease has been recognized as a systemic condition since 2003., ${ }^{5,14}$ Since then, many medical conditions such as Mikulicz's syndrome, Kuttner tumor, and Riedel's thyroiditis which were known as organ-specific disorders are considered as part of IgG4-related disease spectrum. ${ }^{15}$

Immunoglobulin G4-related disease is a chronic relapsing systemic fibroinflammatory disease that can affect any organ with a common key pathological feature including tendency to form inflammatory lesions rich in IgG4-positive plasma cells, storiform fibrosis, phlebitis, and often an elevated IgG4 serum concentration (in $60-70 \%$ of patients). ${ }^{16}$

The prevalence of IgG4-related disease in males is significantly higher than females except for that of the head and neck involvement (such as sialadenitis) which is seen in males and females equally. This gender distribution is in contrast to other autoimmune diseases that mimic IgG4-related disease. ${ }^{17}$ In our study, IgG4-related disease with head and neck involvement occurred in a middle-aged male and a young female that is consistent with the above mentioned gender predominance.

Almost any organ may be affected by IgG4-related disease, usually in the middleaged and elderly patients. ${ }^{18}$ IgG4-related disease symptoms are referable to the specific target organ that may be different from those of other organs. ${ }^{6,17,19}$ IgG4-related disease appears as a mass lesion or diffuse enlargement of organ in most cases which develops gradually and comes to attention usually due to the organ swelling. . $17,20,21$ as observed in the head and neck region with orbital and parotid swelling and neck lymphadenopathy in our patients. Multi-organ involvement occurs in about $60-90 \%$ of patients. ${ }^{22}$ Lymphadenopathy in IgG4-related disease is common (40\%) and mediastinal, hilar, intraabdominal, and axillary lymph nodes are the most commonly involved. ${ }^{21}$ In our patients, clinical features of disease were limited to the head and neck and there was no multi-organ involvement at diagnosis. Hilar adenopathy was observed only in our second patient.

In the head and neck region, various organs including skin, ${ }^{23}$ thyroid, ${ }^{24,25}$ paranasal sinuses, ${ }^{26}$ ears, ${ }^{27,28}$ orbit, ${ }^{29,30}$ and salivary glands may be involved. ${ }^{31}$ The most commonly involved organs by IgG4-related disease in the head and neck region are lacrimal and salivary glands (40\%). ${ }^{15,31,32}$ 
Bilateral or unilateral simultaneous swelling of lacrimal, parotid and salivary glands (previously known as Miculicz disease) and chronic sclerosing sialadenitis (known as Kuttner tumor in the past) were previously known as subtypes of SS. ${ }^{4,16,33-36}$ Both of the above mentioned disorders are now considered as part of the spectrum of IgG4-related disease. ${ }^{31}$ IgG4-related disease can be differentiated from SS by lower rate of xerophthalmia, xerostomia and arthralgias as well as higher rate of allergic rhinitis, bronchial asthma, autoimmune pancreatitis, and interstitial nephritis. ${ }^{31,36-38}$ Low rate of autoantibodies, including rheumatoid factor, antinuclear antibodies, anti-Sjögren'ssyndrome-related antigen A, anti-Sjögren'ssyndrome-related antigen $\mathrm{B}$, higher serum IgG4 and immunoglobulin $\mathrm{E}$ concentrations and better response to steroid therapy are other findings that can help differentiating IgG4-related disease from SS. ${ }^{31,36-38}$ These differences enabled us to rule out Kuttner tumor in our first patient and Miculicz disease in our second patient.

Although the diagnosis of IgG4-related disease is based on the combination of clinical feature, serology, imaging, histopathology and immunohistochemistry findings, histopathology is the gold standard and key component for diagnosing IgG4-related disease. ${ }^{39,40}$

The unique histopathological characteristics of IgG4-related disease include three major features of dense lymphoplasmacytic infiltrate, storiform fibrosis, and phlebitis. ${ }^{16,40}$ Based on the guidelines developed and approved in 2011 by experts in "Consensus statement on the pathology of IgG4-related disease", presence of at least two of the three major histological features is required for definitive histological diagnosis of IgG4-related disease. However, it should be considered that storiform fibrosis and obliterative phlebitis may be absent or non-significant in organs such as the lymph nodes ${ }^{41}$ as well as salivary and lacrimal glands. ${ }^{30}$

Increased number of IgG4+ plasma cells or an elevated ratio of $\mathrm{IgG} 4+/ \mathrm{IgG}+$ in the tissue are of diagnostic value in immunostaining, although the latter can be a more powerful evidence $e^{41,42}$ since infiltration of IgG4+ plasma cells may be seen in some non IgG4-related disease inflammatory lesions. ${ }^{43}$
Elevated serum IgG4 concentration ( $>135 \mathrm{mg} / \mathrm{dL}$ ) is another characteristic of the disease, although serum IgG4 concentration may be normal in $30-40 \%$ of patients with histopathologically confirmed IgG4-related disease. ${ }^{44}$ Some of the specific histopathological and immunostaining characteristics such as lymphoplasmacytic infiltration, fibrosis and infiltration of IgG4+ plasma cells as well as elevated serum IgG4 concentration were observed in both of our patients with head and neck involvement.

The main differential diagnosis of IgG4-related disease is SS. In histopathologic examination, both of these disorders show marked lymphocytic infiltration but IgG4-related disease can be differentiated from SS by lymphoid follicles formation, lower lymphocytic infiltration and marked IgG4+ plasma cells infiltration (>10 IgG4+ cells/HPF) with a ratio of IgG4+ to IgG+ cells of more than $40 \%$, which is never seen in SS. Also, polyclonal B-cell proliferation is seen only in IgG4-related disease but not in SS.9,16,17,34-36,45,46

Kuttner tumor and Mikulicz's syndrome, which were observed in our patients, are two similar disorders that both share a common feature of sialadenitis and now are considered as IgG4-related disease. ${ }^{17}$ Histology of Kuttner tumors shows severe fibrosclerotic lesions with infiltration of IgG4+ plasma cells ${ }^{9}$ but fibrosis is less severe in Mikulicz's syndrome. Therefore, it is difficult to differentiate them from each other. ${ }^{17}$ In our patients, differential diagnosis of Kuttner tumor and Mikulicz's syndrome from IgG4-related disease was challenging and highly elevated serum IgG4 guided us to diagnose IgG4-related disease in the head and neck region.

Magnetic resonance imaging may be beneficial in diagnosis of IgG4-related disease with head and neck involvement. Imaging findings of IgG4-related disease differ based on involved organ. Imaging of IgG4-related disease in head and neck lesions such as lacrimal gland, orbital mass and pituitary reveals hypointense signal in $\mathrm{T}_{2}$ of $\mathrm{MRI},{ }^{47}$ which was observed in MRI of our second patient. So, the diagnosis of IgG4-related disease should be suggested for $\mathrm{T}_{2}$-weighted hypointense enhancing lesions of the head and neck such as lacrimal glands, as in our second patient, and further investigations should be performed. However, 
making a definitive diagnosis by imaging findings alone is challenging.

Glucocorticoids are the treatment of choice and the first line of therapy in IgG4-related disease and most of patients have good response to this treatment except for those with extensive fibrosis. ${ }^{48,49}$ Good response to glucocorticoids is so remarkable that it may further confirm the diagnosis and even help to discriminate it from other similar diseases such as SS..$^{15,31,36-38}$

Other medications such as methotrexate, azathioprine or mycophenolate mofetil may be used to reduce adverse effects of long-term steroids usage. In addition, recent studies have shown significant efficacy of B cell depletion therapy with rituximab in refractory patients. ${ }^{50,51}$

In conclusion, further histopathological, imaging and pharmacological evidence should be provided for better understanding and clarifying various aspects of head and neck involvement in IgG4-related disease. Currently, it is not possible to establish a definitive diagnosis of head and neck involvement in IgG4-related disease by only one of the mentioned methods although its diagnosis is similar to involvement of other organs. A combination of histopathological, immunohistochemistry, imaging and serologic studies should be considered for distinct diagnosis of IgG4-related disease in the head and neck region.

\section{Declaration of conflicting interests}

The authors declared no conflicts of interest with respect to the authorship and/or publication of this article.

\section{Funding}

The authors received no financial support for the research and/or authorship of this article.

\section{REFERENCES}

1. Cravedi P, Abbate M, Gagliardini E, Galbusera M, Buelli S, Sabadini E, et al. Membranous nephropathy associated with IgG4-related disease. Am J Kidney Dis 2011;58:272-5.

2. Okazaki K, Uchida K, Miyoshi H, Ikeura T, Takaoka M, Nishio A. Recent concepts of autoimmune pancreatitis and IgG4-related disease. Clin Rev Allergy Immunol 2011;41:126-38.

3. Nomura $\mathrm{Y}$, Naito $\mathrm{Y}$, Eriguchi $\mathrm{N}$, Kume $\mathrm{T}$, Itai $\mathrm{N}$, Sonoda $\mathrm{H}$, et al. A case of IgG4-related sclerosing mesenteritis. Pathol Res Pract 2011;207:518-21.

4. Fragoulis GE, Moutsopoulos HM. IgG4 syndrome: old disease, new perspective. $J$ Rheumatol 2010;37:1369-70.

5. Kamisawa T, Funata N, Hayashi Y, Eishi Y, Koike $\mathrm{M}$, Tsuruta $\mathrm{K}$, et al. J Gastroenterol. A new clinicopathological entity of IgG4-related autoimmune disease 2003;38:982-4.

6. Khosroshahi A, Stone JH. A clinical overview of IgG4-related systemic disease. Curr Opin Rheumatol 2011;23:57-66.

7. Stone $\mathrm{JH}$, Khosroshahi A, Hilgenberg A, Spooner A, Isselbacher EM, Stone JR. IgG4-related systemic disease and lymphoplasmacytic aortitis. Arthritis Rheum 2009;60:3139-45.

8. Stone JH, Caruso PA, Deshpande V. Case records of the Massachusetts General Hospital. Case 24-2009. A 26-year-old woman with painful swelling of the neck. N Engl J Med 2009;361:511-8.

9. Dahlgren M, Khosroshahi A, Nielsen GP, Deshpande $\mathrm{V}$, Stone JH. Riedel's thyroiditis and multifocal fibrosclerosis are part of the IgG4-related systemic disease spectrum. Arthritis Care Res (Hoboken) 2010;62:1312-8.

10. Zhang L, Smyrk TC. Autoimmune pancreatitis and IgG4-related systemic diseases. Int J Clin Exp Pathol 2010;3:491-504.

11. Kamisawa T, Takuma K, Egawa N, Tsuruta K, Sasaki T. Autoimmune pancreatitis and IgG4-related sclerosing disease. Nat Rev Gastroenterol Hepatol 2010;7:401-9.

12. Liu Y, Sun YC, Feng RE, Liu XF, Liu GJ, Liu HG. IgG4-related lung disease: a case report and review of the literature. Zhonghua Jie $\mathrm{He} \mathrm{He} \mathrm{Hu} \mathrm{Xi} \mathrm{Za}$ Zhi 2012;35:752-7.

13. Griepentrog GJ, Vickers RW, Karesh JW, Azari AA, Albert DM, Bukat CN. A clinicopathologic case study of two patients with pediatric orbital IgG4-related disease. Orbit 2013;32:389-91.

14. Kamisawa $\mathrm{T}$, Egawa $\mathrm{N}$, Nakajima $\mathrm{H}$. Autoimmune pancreatitis is a systemic autoimmune disease. Am J Gastroenterol 2003;98:2811-2.

15. Guma M, Firestein GS. IgG4-related diseases. Best Pract Res Clin Rheumatol 2012;26:425-38.

16. Masaki Y, Dong L, Kurose N, Kitagawa K, Morikawa Y, Yamamoto M, et al. Proposal for a new clinical entity, IgG4-positive multiorgan lymphoproliferative syndrome: analysis of 64 cases of IgG4-related disorders. Ann Rheum Dis 2009;68:1310-5.

17. Umehara H, Okazaki K, Masaki Y, Kawano M, Yamamoto M, Saeki T, et al. A novel clinical entity, IgG4-related disease (IgG4RD): general concept and details. Mod Rheumatol 2012;22:1-14.

18. Zen $\mathrm{Y}$, Inoue $\mathrm{D}$, Kitao $\mathrm{A}$, Onodera $\mathrm{M}$, Abo $\mathrm{H}$, Miyayama S, et al. IgG4-related lung and pleural disease: a clinicopathologic study of 21 cases. Am J Surg Pathol 2009;33:1886-93.

19. Cheuk W, Chan JK. IgG4-related sclerosing disease: a critical appraisal of an evolving clinicopathologic entity. Adv Anat Pathol 2010;17:303-32. 
20. Zen Y, Nakanuma Y. IgG4-related disease: a crosssectional study of 114 cases. Am J Surg Pathol 2010;34:1812-9

21. Saegusa H, Momose M, Kawa S, Hamano H, Ochi Y, Takayama M, et al. Hilar and pancreatic gallium-67 accumulation is characteristic feature of autoimmune pancreatitis. Pancreas 2003;27:20-5.

22. Okazaki K, Uchida K, Koyabu M, Miyoshi H, Takaoka M. Recent advances in the concept and diagnosis of autoimmune pancreatitis and IgG4-related disease. J Gastroenterol 2011;46:277-88.

23. Ikeda T, Oka M, Shimizu H, Hatakeyama M, Kanki $\mathrm{H}$, Kunisada $\mathrm{M}$, et al. IgG4-related skin manifestations in patients with IgG4-related disease. Eur J Dermatol 2013;23:241-5.

24. Li Y, Nishihara E, Kakudo K. Hashimoto's thyroiditis: old concepts and new insights. Curr Opin Rheumatol 2011;23:102-7.

25. Li Y, Nishihara E, Hirokawa M, Taniguchi E, Miyauchi A, Kakudo K. Distinct clinical, serological, and sonographic characteristics of hashimoto's thyroiditis based with and without IgG4-positive plasma cells. J Clin Endocrinol Metab 2010;95:1309-17.

26. Ikeda R, Awataguchi T, Shoji F, Oshima T. A case of paranasal sinus lesions in IgG4-related sclerosing disease. Otolaryngol Head Neck Surg 2010;142:458-9.

27. Cho HK, Lee YJ, Chung JH, Koo JW. Otologic Manifestation in IgG4-Related Systemic Disease. Clin Exp Otorhinolaryngol 2011;4:52-4.

28. Takagi D, Nakamaru Y, Fukuda S. Otologic manifestations of immunoglobulin G4-related disease. Ann Otol Rhinol Laryngol 2014;123:420-4.

29. Sato Y, Ohshima K, Ichimura K, Sato M, Yamadori I, Tanaka T, et al.Pathol Int. Ocular adnexal IgG4-related disease has uniform clinicopathology 2008;58:465-70.

30. Cheuk W, Yuen HK, Chan JK. Chronic sclerosing dacryoadenitis: part of the spectrum of IgG4-related Sclerosing disease? Am J Surg Pathol 2007;31:643-5.

31. Moutsopoulos HM, Fragoulis GE, Stone JH. Overview of IgG4-related disease. Uptodate 2014.

32. Hamano H, Arakura N, Muraki T, Ozaki Y, Kiyosawa K, Kawa S. Prevalence and distribution of extrapancreatic lesions complicating autoimmune pancreatitis. J Gastroenterol 2006;41:1197-205.

33. Himi T, Takano K, Yamamoto M, Naishiro Y, Takahashi H. A novel concept of Mikulicz's disease as IgG4-related disease. Auris Nasus Larynx 2012;39:9-17.

34. Masaki Y, Sugai S, Umehara H. IgG4-related diseases including Mikulicz's disease and sclerosing pancreatitis: diagnostic insights. J Rheumatol 2010;37:1380-5.

35. Yamamoto M, Takahashi H, Ohara M, Suzuki C, Naishiro Y, Yamamoto H, et al. A new conceptualization for Mikulicz's disease as an IgG4-related plasmacytic disease.Mod Rheumatol 2006;16:335-40.

36. Yamamoto M, Takahashi H, Sugai S, Imai K. Clinical and pathological characteristics of Mikulicz's disease (IgG4-related plasmacytic exocrinopathy). Autoimmun Rev 2005;4:195-200.
37. Takahashi H, Yamamoto M, Tabeya T, Suzuki C, Naishiro Y, Shinomura Y, et al. The immunobiology and clinical characteristics of IgG4 related diseases. $\mathrm{J}$ Autoimmun 2012;39:93-6.

38. Yamashita K, Haga H, Mikami Y, Kanematsu A, Nakashima $\mathrm{Y}$, Kotani $\mathrm{H}$, et al. Degree of IgG4+ plasma cell infiltration in retroperitoneal fibrosis with or without multifocal fibrosclerosis. Histopathology 2008;52:404-9.

39. Deshpande V. The pathology of IgG4-related disease: critical issues and challenges. Semin Diagn Pathol 2012;29:191-6.

40. Deshpande V, Zen Y, Chan JK, Yi EE, Sato Y, Yoshino $\mathrm{T}$, et al. Consensus statement on the pathology of IgG4-related disease. Mod Pathol 2012;25:1181-92.

41. Cheuk W, Yuen HK, Chu SY, Chiu EK, Lam LK, Chan JK. Lymphadenopathy of IgG4-related sclerosing disease. Am J Surg Pathol 2008;32:671-81.

42. Sato Y, Notohara K, Kojima M, Takata K, Masaki Y, Yoshino T. IgG4-related disease: historical overview and pathology of hematological disorders. Pathol Int 2010;60:247-58.

43. Sato Y, Kojima M, Takata K, Morito T, Asaoku $\mathrm{H}$, Takeuchi $\mathrm{T}$, et al. Systemic IgG4-related lymphadenopathy: a clinical and pathologic comparison to multicentric Castleman's disease. Mod Pathol 2009;22:589-99.

44. Sah RP, Chari ST. Serologic issues in IgG4-related systemic disease and autoimmune pancreatitis. Curr Opin Rheumatol 2011;23:108-13.

45. Yamamoto M, Takahashi H, Hasebe K, Suzuki $\mathrm{C}$, Naishiro Y, Hayashi $\mathrm{T}$, et al. The analysis of interleukin-6 in patients with systemic IgG4-related plasmacytic syndrome--expansion of SIPS to the territory of Castleman's disease. Rheumatology (Oxford) 2009;48:860-2.

46. Yamamoto M, Takahashi H, Ohara M, Suzuki C, Naishiro Y, Yamamoto H, et al. A case of Mikulicz's disease (IgG4-related plasmacytic disease) complicated by autoimmune hypophysitis. Scand J Rheumatol 2006;35:410-1.

47. Toyoda K, Oba H, Kutomi K, Furui S, Oohara A, Mori $\mathrm{H}$, et al. MR imaging of IgG4-related disease in the head and neck and brain.AJNR Am J Neuroradiol 2012;33:2136-9.

48. Stone JH, Zen Y, Deshpande V. IgG4-related disease. N Engl J Med 2012;366:539-51.

49. Khosroshahi A, Stone JH. Treatment approaches to IgG4-related systemic disease. Curr Opin Rheumatol 2011;23:67-71.

50. Khosroshahi A, Carruthers MN, Deshpande V, Unizony $\mathrm{S}$, Bloch DB, Stone JH. Rituximab for the treatment of IgG4-related disease: lessons from 10 consecutive patients. Medicine (Baltimore) 2012;91:57-66.

51. Sedyshev SKh, Vasil'ev VI, Kovrigina AM, Logvinenko OA, Rodionova EB, Safonova TN, et al. IgG4-related disease: patient group characterization and rituximab therapy.Ter Arkh 2013;85:48-53. 